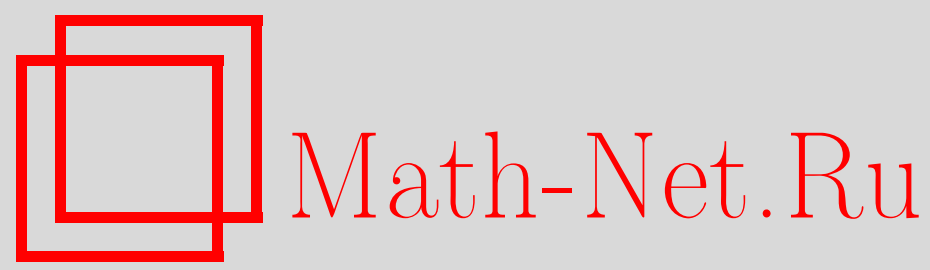

П. В. Андреев, Об определимых предикатах стандартности в теории внутренних множеств, Матем. заметки, 1999, том 66, выпуск 6, 803-809

DOI: https://doi.org/10.4213/mzm1225

Использование Общероссийского математического портала Math-Net.Ru подразумевает, что вы прочитали и согласны с пользовательским соглашением http://www . mathnet.ru/rus/agreement

Параметры загрузки:

IP: 52.23 .180 .231

26 апреля 2023 г., 09:50:22

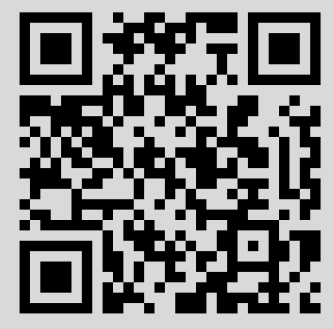




\title{
ОБ ОПРЕДЕЛИМЫХ ПРЕДИКАТАХ СТАНДАРТНОСТИ В ТЕОРИИ ВНУТРЕННИХ МНОЖЕСТВ
}

\section{П. В. Андреев}

\begin{abstract}
Доказьвается, что в IST - теории внутренних множеств Нельсона - не существует определимого предиката, являюшегося собственным расширением предиката стандартности и удовлетворяющего принципам переноса, идеализации и стандартизации.

Библиография: 15 названий.
\end{abstract}

В различных аксиоматиках нестандартной теории множеств из универсума всех множеств выделяется собственньй подкласс стандартных множеств. Это осуществляется либо посредством добавления специального одноместного предиката st к обычному языку формальной теории множеств и формулированием аксиом, описьвающих свойства этого предиката, либо этот класс определяется средствами теории множеств, а его свойства доказьваются в соответствующей формальной системе. Первый подход представлен аксиоматическими системами Нельсона, Хрбачека, Каваи и Кановея. Подробный обзор содержится в монографии [1], где также обсуждаются математические и философские представления, лежащие в основе свойств, характеризуюших стандартные множества. Второй подход применяется в альтернативной теории множеств П. Вопенки [2], а также в работе [3].

Наиболее распространенной в настоящее время аксиоматической системой нестандартной теории множеств является теория внутренних множеств Э. Нельсона IST (см. [4]), которая получена добавлением к языку теории множеств Цермело-Френкеля одноместного предиката стандартности st, a к системе аксиом ZFC трех дополнительных принципов (схем аксиом).

ПЕРенос Т. $\exists x \phi(x) \longrightarrow \exists^{\text {st }} x \phi(x)$, где $\phi-$ внутренняя формула со стандартньми параметрами.

ИдЕАЛИЗАЦИЯ I. $\forall^{\text {st fin }} A \exists x \forall a \in A \phi(x, a) \longleftrightarrow \exists x \forall^{\text {st }} a \phi(x, a)$, где $\phi$-внутренняя формула.

Работа выполнена при поддержке Российского фонда фундаментальных исследований, грант № 98-01-00045. 
СТАНДАРТИЗАЦИя S. $\forall^{\mathrm{st}} X \exists^{\mathrm{st}} Y \forall^{\mathrm{st}} z(x \in Y \longleftrightarrow x \in X \& \Phi(x))$, где $\Phi$ - произвольная формула языка IST.

Здесь запись вида $\forall^{\text {st }} x \Psi$, где $\Psi$ - формула язька IST, есть сокрашение для формулы $\forall x($ st $x \longrightarrow \Psi) ; \forall^{\text {st fin }} x \Psi$ есть сокращение для $\forall x$ (st $x \&$ fin $x \longrightarrow \Psi$ ), где fin $x$, в свою очередь, обозначает формулу, выражающую свойство $x$ быть конечньг множеством. Аналогично запись $\exists^{\text {st }} x \Psi$ есть сокращение для формулы $\exists x$ (st $x \& \Psi$ ). Формула назьвается внутренней, если она не содержит предиката st.

В связи с исследованием аксиоматических систем нестандартной теории множеств и, в частности, вопросов независимости представляет интерес изучение определимых в этих системах классов, являющихся собственными расширениями класса стандартных множеств и обладающих различными свойствами последнего. Так, например, в работе [5] построено семейство определимых в IST классов, каждый из которых является расширением класса стандартных множеств и удовлетворяет аксиомам переноса и идеализации. Там же доказано существование класса из этого семейства, для которого не выполнен принцип стандартизации. Таким образом, была доказана независимость аксиом стандартизации от остальных аксиом IST. Упомянутое семейство классов задается двуместным рефлексивным транзитивным предикатом относительной стандартности $\cdot$ st $\cdot$, определяемым формулой

$$
x \text { st } y \stackrel{\text { def }}{\longleftrightarrow} \exists^{\text {st }} f \in \text { Fnc }(\text { fin } f(x) \& y \in f(x)),
$$

где $x$ и $y$ - произвольные множества, a Fnc - класс всех функций. Каждьй класс семейства имеет вид $\mathbb{S}_{\tau}=\{x: x$ st $\tau\}$, где $\tau$ есть некоторое ограниченное (содержащееся в известном стандартном) множество.

Изначально исследование предиката относительной стандартности было стимулировано часто возникающей в нестандартном анализе необходимостью в рассмотрении нестандартных объектов аналогично стандартным, в перенесении понятий и техники, применяемых для стандартных объектов, на нестандартные.

Примеры использования предиката относительной стандартности в IST можно найти в книге [6]. В рамках робинсоновского нестандартного анализа соответствующая техника развита в работах [7]-[9], где приведены многочисленные применения этой техники (см. также [10]). В альтернативной теории множеств было введено понятие сдвигов горизонта (см. [11]).

Нарушение принципа стандартизации для предиката относительной стандартности Е. И. Гордона ограничивает возможности его применения для исследования нестандартных объектов в IST. В теории RIST И. Перэра (см. [12]) аксиоматически вводится рефлексивный транзитивный предикат относительной стандартности $\cdot \mathcal{S R} \cdot$. При этом для любых множеств $x$ и $y$ либо $x \mathcal{S R} y$, либо $y \mathcal{S R} x$, и каждьй класс вида $\mathrm{SR}_{\alpha}=\{x: x \mathcal{S R} \alpha\}$ подчиняется принципам переноса, идеализации и стандартизации. Роль класса стандартных множеств в этой теории вьполняет класс $\mathrm{SR}_{0}=\{x: \forall \alpha$ $(x \mathcal{S R} \alpha)\}$. В связи с этим возникает вопрос о возможности определения предиката со свойствами, подобньги свойствам предиката $\mathcal{S} \mathcal{R}$, формулой IST.

В. Г. Кановей высказал предположение о том, что ответ на этот вопрос отрицательный. Он сформулировал более общую гипотезу: не существует определимого в IST предиката, расширяющего “обьчньй” предикат стандартности и обладающего всеми его 
свойствами. В настоящей работе эта гипотеза доказьвается при некоторых дополнительных условиях.

Пусть std - одноместный предикат, определимый в IST, т.е. $\operatorname{std} x \stackrel{\text { def }}{\longleftrightarrow} \Phi(x)$, где $\Phi-$ формула языка IST с параметрами. Пусть $\Psi$ есть некоторое предложение языка IST, возможно, с параметрами. Будем говорить, что предикат std yдовлетворяет $\Psi$, если истинно предложение $\Psi[\mathrm{std}]$, полученное заменой всех вхождений st в $\Psi$ на std.

Утверждение, котороемы докажем, теперь можно сформулировать так: не существует определимого в IST предиката, являющегося собственньг расширением предиката стандартности st и удовлетворяющего принципам переноса, идеализации и стандартизации. Упомянутые выше дополнительные условия таковы.

Во-первых, доказательство проводится в теории BST (см. [13], [14]), которая аксиоматизирует класс $\left\{x: \exists^{\text {st }} X(x \in X)\right\}$ ограниченных множеств теории IST. Эта теория удобна для приложений, поскольку классы в BST имеют регулярную структуру. Теорема остается справедливой и в IST, если формула, задающая “новый” предикат стандартности std, является ext-ограниченной (содержит st только в составе кванторов вида $\exists^{\text {st }} x \in X, \forall^{\text {st }} x \in X$, где $X$ - стандартное множество) и имеет ограниченные параметры.

Теория BST имеет тот же язык и содержит те же аксиомы, что и IST, за исключением идеализации $\mathbf{I}$, которая заменена на ограниченную идеализацию, и одной дополнительной аксиомы ограниченности.

ОГРАНИЧЕННАЯ ИДЕАЛИЗАЦИЯ ВІ. $\forall^{\text {st fin }} A \subseteq A_{0} \exists x \forall a \in A \phi(x, a) \longleftrightarrow \exists x$ $\forall^{\mathrm{st}} a \in A_{0} \phi(x, a)$, где $\phi$ - внутренняя формула и $A_{0}$ - произвольное стандартное множество.

\section{ОГРАНИЧЕНность В. $\forall x \exists^{\mathrm{st}} X(x \in X)$.}

Понятия и соглашения, принятые нами для IST, остаются в силе и для BST. Cmaндартным приближением ${ }^{\mathrm{s}} \mathbf{X}$ класса $\mathbf{X}$ назовем стандартное множество, содержащее те же стандартные элементы, что и $\mathbf{X}$. Таким образом, принцип стандартизации утверждает наличие стандартного приближения для всякого класса, включенного в некоторое стандартное множество. Через ${ }^{\circ} \mathbf{X}$ будем обозначать класс $\{x \in \mathbf{X}:$ st $x\}$ стандартных элементов X.

Вторьм дополнительньм условием является предположение о существовании stdстандартного, но нестандартного натурального числа. Следующее предложение показьвает, что это условие не является сильньм ограничением.

ПРЕДЛОЖЕНИЕ 1 (BST). Пусть $x$ есть нестандартное множество такое, что для всякой стандартной функиии $f$, содержащей $x$ в области определения, множество $f(x)$ стандартно, если $f(x) \in \omega$. Тогда всякое стандартное множество $X \ni x$ имеет мощность, которая является измеримым кардиналом.

Если предикат std расширяет st и удовлетворяет принципу переноса, то для любого $x$ и любой стандартной функции $f$ из std $x$ следует std $f(x)$. Поэтому если не сушествует std-стандартных нестандартных натуральных чисел, то по предложению 1 std совпадает c st на элементах любого множества неизмеримой мощности. 
ДОКАЗАТЕЛЬСТВО ПРЕДЛОЖЕНИЯ 1 . Пусть $X \ni x$-стандартное множество. Положим $U={ }^{s}\{Y \subseteq X: x \in Y\}$. По принципу переноса $U$ является неглавньпм ультрафильтром на $X$. Пусть $\left\{A_{i}: i \in \omega\right\}$ - произвольное стандартное счетное разбиение $X$. Оно задает функцию $f: X \rightarrow \omega$ по правилу $f(y)=i \longleftrightarrow y \in A_{i}$. По условию для некоторого стандартного $i_{0} \in \omega$ имеем $f(x)=i_{0}$. Согласно принципу переноса множество $A_{i_{0}}$ стандартно. Кроме того, $A_{i_{0}} \in U$ по выбору $f$ и $U$. Снова применяя принцип переноса, получаем, что всякое счетное разбиение множества $X$ имеет член, принадлежащий $U$. Поэтому ультрафильтр $U$ является счетно полньм, и мощность $X$ измерима.

Ниже формулу $\Psi$ будем называть std- $\in$-формулой, ecли она содержит предикат st только в составе подформул вида std $x$. Классы, определяемые std- $\epsilon$-формулами, будем назьвать std- $\epsilon$-определимыми.

ТЕОРема 1 (BST). Пусть определимый одноместный предикат std обладает следующими свойствами:

1) $\forall x($ st $x \longrightarrow \operatorname{std} x)$;

2) std-перенос: $\forall^{\text {std }} x \phi(x) \longrightarrow \forall x \phi(x)$ для любой внутренней формуль ф $с$ std-стандартными параметрами;

3) std-идеализация: $\forall^{\text {std }} A_{0}\left(\forall^{\text {std fin }} A \subseteq A_{0} \exists x \forall a \in A \phi(x, a) \longleftrightarrow \exists x \forall^{\text {std }} a \in A_{0}\right.$ $\phi(x, a))$ для внутренних формул ф с произвольными параметрами;

4) $\exists n \in \omega(\operatorname{std} n \& \neg \operatorname{st} n)$.

Тогда существует мнохество $X$ такое, что

$$
\neg \exists^{\mathrm{std}} Y \forall^{\mathrm{std}} x(x \in X \longleftrightarrow x \in Y) .
$$

ДоказАТЕЛьСтво. Сечением в множестве натуральных чисел называется класс $\mathbf{C} \subseteq \omega$, для которого из $n \in \mathbf{C}$ следует $n \subseteq \mathbf{C}$. Для любого класса $\mathbf{X} \subseteq \omega$ через $\widetilde{\mathbf{X}}$ будем обозначать наименьшее сечение, содержащее $\mathbf{X}$.

Положим $\operatorname{Std}_{\omega}=\{k \in \omega: \operatorname{std} k\}$. Доказательство в BST того факта, что все элементы любого стандартного конечного множества стандартны, использует только принцип переноса и принцип идеализации (а именно, импликацию справа налево; см. [13]), поэтому соответствующий факт имеет место и для предиката std. Отсюда следует, что $\operatorname{Std}_{\omega}$ есть сечение, причем по std-идеализации $\operatorname{Std}_{\omega} \neq \omega$. По свойству std-переноса $\operatorname{Std}_{\omega}$ не может быть множеством.

Сначала мы покажем, что $\operatorname{Std}_{\omega}$ является $\Pi_{1}^{\text {st }}$ - или $\Sigma_{1}^{\text {st }}$-классом.

ЛЕмма 1. Имеются только две взаимоисключающие возмохности:

1) $\exists^{\mathrm{st}} B \exists \mu: B \rightarrow \omega\left(\operatorname{Std}_{\omega}=\bigcap_{b \in{ }^{\circ} B} \mu(b)\right)$;

2) $\exists^{\mathrm{st}} A \exists \nu: A \rightarrow \omega\left(\operatorname{Std}_{\omega}=\bigcup_{a \in^{\circ} A} \nu(a)\right)$.

ДокАЗАТЕЛЬСТво. В ВST любой подкласс множества может быть определен ехtограниченной $\Sigma_{2}^{\text {st }}$-формулой (см. [13], [15]). Поэтому $\operatorname{Std}_{\omega}$ представим в виде

$$
\operatorname{Std}_{\omega}=\bigcup_{a \in \in^{\circ} A} \bigcap_{b \in \in^{\circ} B} M_{a b},
$$


где множества $A$ и $B$ стандартны и $M$ есть внутренняя функция двух аргументов. Положим

$$
\mathbf{C}_{a}=\bigcap_{b \in{ }^{\circ} B} M_{a b}
$$

Имеем

$$
\operatorname{Std}_{\omega}=\bigcup_{a \in \in^{\circ} A} \mathbf{C}_{a}=\bigcup_{a \in \in^{\circ} A} \widetilde{\mathbf{C}}_{a}
$$

Для любого $n \in \omega$ с помощью идеализации получаем

$$
\begin{aligned}
n \in \widetilde{\mathbf{C}}_{a} & \longleftrightarrow \exists k>n \forall^{\text {st }} b \in B\left(k \in M_{a b}\right) \\
& \longleftrightarrow \forall^{\text {st fin }} B_{0} \subseteq B \exists k>n \forall b \in B_{0}\left(k \in M_{a b}\right) .
\end{aligned}
$$

Таким образом,

$$
\widetilde{\mathbf{C}}_{a}=\bigcap_{\beta \in{ }^{\circ} \mathcal{B}} \widetilde{M}_{a \beta}^{\prime},
$$

где $\mathcal{B}=\mathcal{P}^{\text {fin }}(B)$ и $M_{a \beta}^{\prime}=\bigcap_{b \in \beta} M_{a b}$.

Возможны два случая.

а) Существует стандартное $a \in A$ такое, что $\forall^{\text {st }} a^{\prime} \in A\left(\widetilde{\mathbf{C}}_{a^{\prime}} \subseteq \widetilde{\mathbf{C}}_{a}\right)$. Тогда $\operatorname{Std}_{\omega}=\widetilde{\mathbf{C}}_{a}$. Предполагая, что $\forall^{\operatorname{std}} \beta \in \mathcal{B}\left(\widetilde{M}_{a \beta}^{\prime} \neq \omega\right)$, и обозначая $\mu(\beta)=\max \widetilde{M}_{a \beta}^{\prime}$, мы получаем представление $\operatorname{Std}_{\omega}=\bigcap_{\beta \in \mathcal{B}} \mu(\beta)$.

б) $\forall^{\text {st }} a \in A \exists^{\text {st }} a^{\prime} \in A\left(\widetilde{\mathbf{C}}_{a} \subsetneq \widetilde{\mathbf{C}}_{a^{\prime}}\right)$. В этом случае мы имеем

$$
\forall^{\mathrm{st}} a \in A \exists^{\mathrm{st}} \beta \in \mathcal{B}\left(\widetilde{M}_{a \beta}^{\prime} \subseteq \operatorname{Std}_{\omega}\right)
$$

Применяя стандартизацию и аксиому выбора, получаем

$$
\exists^{\mathrm{st}} f: A \rightarrow \mathcal{B} \forall^{\mathrm{st}} a \in A\left(\widetilde{M}_{a, f(a)}^{\prime} \subseteq \operatorname{Std}_{\omega}\right)
$$

Следовательно, $\operatorname{Std}_{\omega}=\bigcup_{a \in{ }^{\circ} A} \nu(a)$, где $\nu(a)=\max \widetilde{M}_{a, f(a)}^{\prime}$.

Рассмотрим отдельно каждую из возможностей, описанных в лемме 1 .

1) Пусть $\operatorname{Std}_{\omega}=\bigcap_{b \in{ }^{\circ} B} \mu(b)$ для стандартного множества $B$ и внутренней функции $\mu: B \rightarrow \omega$. Положим $\mathbf{X}=\{b \in B: \neg \operatorname{std} \mu(b)\}$. Очевидно, $\mathbf{X} \supseteq{ }^{\circ} B$. Пусть существует std-стандартное множество $Y$, имеющее те же std-стандартные элементы, что и $\mathbf{X}$. Согласно условию 1 ) теоремы ${ }^{\circ} B \subseteq Y$. Тогда

$$
\forall^{\text {std fin }} K \subseteq \omega \forall^{\text {stdfin }} Y^{\prime} \subseteq Y \exists n \in \omega \forall k \in K \forall y \in Y^{\prime}(k<n<\mu(y)) .
$$

Далее по std-идеализации существует $n \in \omega$ такое, что

$$
\forall^{\text {std }} k \in \omega(n>k) \& \forall^{\text {std }} y \in Y(n<\mu(y)) \text {. }
$$

$\mathrm{C}$ одной стороны, $n$ должно быть std-стандартньг, поскольку ${ }^{\circ} B \subseteq Y$. С другой стороны, $n$ std-бесконечно велико. Это противоречие доказьвает, что $\mathbf{X}$ не имеет stdстандартного приближения. 
В заметке [15] показано, что в теории $\mathbf{B S T}^{-}$(BST без схемы стандартизации) принцип стандартизации $\mathbf{S}$ эквивалентен стандартизации только для внутренних формул. Поэтому, на самом деле, можно найти множество $X$, не имеющее std-стандартного приближения.

2) $\operatorname{Std}_{\omega}=\bigcup_{a \in^{\circ} A} \nu(a)$ для стандартного множества $A$ и внутренней функции $\nu: A \rightarrow \omega$.

Пусть $n_{0} \in \omega$ есть std-стандартное, но нестандартное натуральное число. По принципу идеализации существует строго возрастающая функция $h: n_{0} \rightarrow \omega$ такая, что $\operatorname{ran}\left(\nu \uparrow^{\circ} A\right) \subseteq \operatorname{ran} h$, поскольку такая функция существует, если вместо $A$ взять любое его стандартное конечное подмножество.

Для любого множества $X$ обозначим через $X_{\#}$ функцию, определенную правилом $X_{\#}(n)=\max \{k:|k \cap X|=n\}\left(X_{\#}(n)\right.$ есть $n$-й по возрастанию элемент множества $\left.X\right)$. Таким образом, $X_{\#}$ есть строго возрастающая функция, $\operatorname{dom} X_{\#}=|X|$.

Положим $X=\operatorname{ran} h$. Очевидно, $h=X_{\#}$. Пусть $Y$ есть std-стандартное приближение $X$, т.е.

$$
\operatorname{std} Y \& X \cap \operatorname{Std}_{\omega}=Y \cap \operatorname{Std}_{\omega} .
$$

Нетрудно убедиться в том, что функции $h$ и $Y_{\#}$ совпадают на любом множестве вида $\{n: h(n)<k\}, \operatorname{std} k$ и, следовательно, на классе $\mathbf{X}=\{n: \operatorname{std} h(n)\}$. Класс $Y_{\#}(\mathbf{X})=h(\mathbf{X})$ кофинален с $\operatorname{Std}_{\omega}$ по выбору $h$. Поэтому мы можем записать

$$
\forall^{\mathrm{std}} k \exists n<n_{0}\left(Y_{\#}(n)>k\right) .
$$

По std-идеализации найдется $n<n_{0}$ такое, что $\forall^{\text {std }} k\left(Y_{\#}(n)>k\right)$. Итак, число $Y_{\#}(n)$ является std-бесконечно большим. В то же время, $n$ std-стандартно, так как $\operatorname{Std}_{\omega}$ есть сечение, и, следовательно, по принципу std-переноса $Y_{\#}(n)$ также std-стандартно.

Полученное противоречие показывает, что std-стандартного приближения для $X$ не существует.

Более того, поскольку множество $X$ гиперконечно, мы можем указать рациональное число $r \in(0,1)$, которое не имеет std-стандартной части. Для этого нужно в качестве дробной части двоичной дроби, представляющей $r$, взять индикатор $\chi: \widetilde{X} \rightarrow 2(\chi(n)=1$ $\longleftrightarrow n \in X)$.

Автор благодарит профессора Е. И. Гордона и профессора В. Г. Кановея за интерес к настоящей работе и помощь.

\section{СПИСОК ЦИТИРОВАННОЙ ЛИТЕРАТУРЫ}

[1] Кусраев А. Г., Кутателадзе С. С. Нестандартные методы анализа. Новосибирск: Наука, 1990.

[2] Вопенка П. Математика в алтернативной теории множеств. М.: Мир, 1983.

[3] Ballard D., Hrbáček K. Standard foundations for nonstandard analysis // J. Symbolic Logic. 1992. V. 57. №2. P. 741-748.

[4] Nelson E. Internal set theory: a new approach to nonstandard analysis // Bull. Amer. Math. Soc. (N. S. ). 1977. V. 83. №6. P. 1165-1198. 
[5] Гордон Е. И. Относительно стандартные элементы в теории внутренних множеств Э. Нельсона // Сиб. матем. ж. 1989. Т. 30. №1. С. 89-95.

[6] Gordon E. I. Nonstandard Methods in Commutative Harmonic Analysis. Providence (R.I.): Amer. Math. Soc., 1997.

[7] Beninghofen B., Richter M. A general theory of superinfinitesimals // Fund. Math. 1987. V. 123. № 3. P. 199-215.

[8] Stroyan K., Beninghofen B., Richter M. Superinfinitesimals in topology and functional analysis // Proc. London Math. Soc. (3). 1989. V. 5. № 1. P. 153-181.

[9] Молчанов В. А. О применении повторных нестандартных расширений в топологии // Сиб. матем. ж. 1989. Т. 30. №3. С. 64-71.

[10] Гордон Е. И. Относительная стандартность и $\pi$-монады Бенингхофена-Рихтера // Оптимизация. 1990. № $48(65)$.

[11] Sochor A., Vopěnka P. Shiftings of the horizon // Comment. Math. Univ. Carolin. 1983. V. 24. P. 127-136.

[12] Peraire Y. Théorie relative des ensembles internes // Osaka J. Math. 1992. V. 29. P. 267-297.

[13] Кановей В. Г. Неразрешимые гипотезы в теории внутренних множеств Эдварда Нельсона // УМH. 1991. T. 46. №6. C. 3-50.

[14] Kanovei V., Reeken M. Internal approach to external sets and universes. Part 1. Bounded set theory // Studia Logica. 1995. V. 55. № 2. P. 229-257.

[15] Андреев П. В. О стандартизации в теории ограниченных множеств // Вестн. МГУ. Сер. 1. Матем., мех. 1997. №1. С. 68-70.

Нижегородский государственньй университет

Поступило

E-mail : viron@redline.ru 